\title{
Elastografía en tiempo real (Supersonic): comportamiento de la prueba en una cohorte de pacientes con cirrosis en un centro médico de Bogotá
}

\author{
Real-time shearwave elastography (SSWE) in a cohort of patients with \\ cirrhosis at a medical center in Bogotá
}

Jhon E. Prieto 0., ${ }^{1}$ Nathaly Garzón-Orjuela, ${ }^{2}$ Santiago Sánchez P. , ${ }^{3}$ Robin G. Prieto 0., ${ }^{1}$ Andrés F. Ochoa D., ${ }^{3}$ Javier Eslava-Schmalbach. ${ }^{2}$

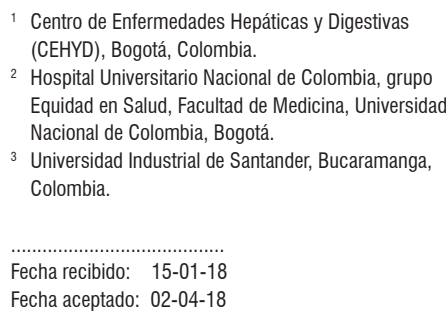

3 Universidad Industrial de Santander, Bucaramanga, Colombia.

Fecha recibido: $\quad 15-01-18$

Fecha aceptado: 02-04-18

\begin{abstract}
Resumen
Introducción: la cirrosis, entendida como un proceso dinámico y reversible, es el resultado final de las enfermedades hepáticas crónicamente progresivas de diferentes etiologías. Los métodos no invasivos basados en ecografía se han convertido paulatinamente en una herramienta diagnóstica útil en el estudio de las hepatopatías. Dentro de estos métodos, la elastografía en tiempo real, o Supersonic, de reciente introducción en nuestro medio, nos permite estudiar estos pacientes. Objetivo: evaluar el comportamiento de la elastografía en tiempo real en pacientes con cirrosis en un centro de atención de enfermedades hepáticas y digestivas en la ciudad de Bogotá. Materiales y métodos: estudio piloto retrospectivo de una cohorte con pacientes cirróticos con diagnóstico comprobado por biopsia hepática, imágenes, clínica o una combinación de estas. Resultados: se incluyeron 65 pacientes diagnosticados con cirrosis, la mediana de edad fue 58 años, $61,5 \%$ mujer y $38,5 \%$ hombre. Se encontró una mediana de rigidez hepática de 16,6 kilopascal (kPs) (RIQ de 13,9-19,5), con valor mínimo de 12 y un máximo de 30,5; sin una diferencia estadísticamente significativa entre mujeres y hombres dentro de los grupos con y $\sin$ biopsia ( $p=0,64$ y $p=0,26$, respectivamente). Conclusión: los valores de la rigidez hepática de los pacientes cirróticos analizados en este estudio piloto se encontraron dentro de los reportados en los estudios iniciales de las pruebas a nivel internacional, por lo que se podría sugerir la realización de esta prueba no invasiva en pacientes con sospecha de cirrosis, evitando así las complicaciones de la biopsia hepática.
\end{abstract}

Palabras clave

Elastografía en tiempo real, Supersonic, fibrosis hepática, cirrosis.

\begin{abstract}
Introduction: Cirrhosis, understood as a dynamic and reversible process, is the final result of chronically progressive liver diseases of various etiologies. Non-invasive methods based on ultrasound have gradually become useful diagnostic tools for studying liver diseases. Real-time supersonic shearwave elastography (SSWE) study of these patients has recently been introduced to Colombia. Objective: The objective of this study was to evaluate real-time SSWE in patients with cirrhosis at a center for the treatment of liver and digestive diseases in Bogotá. Materials and Methods: This is a retrospective pilot study of a cohort of cirrhotic patients with diagnoses confirmed by liver biopsies, imaging, clinical indicators or a combination of these. Results: Sixty-five patients who had been diagnosed with cirrhosis were included in this study. The median age was 58 years, $61.5 \%$ were women, and $38.5 \%$ were men. We found that median hepatic stiffness was 16.6 kilopascals $(\mathrm{kPs})$, the interquartile range was $13.9-19.5$, the minimum value was 12 , and the maximum value was 30.5 . There were no statistically significant differences between women and men, within groups and with and without biopsies ( $p=0.64$ and $p=0.26$ respectively). Conclusions: Hepatic rigidity measurements for cirrhotic patients analyzed in this pilot study were within the ranges reported in initial international tests, so this non-invasive test should be considered for patients suspected of having cirrhosis as a way to avoid complications of liver biopsies.
\end{abstract}

Keywords

Real-time elastography, supersonic, liver fibrosis, cirrhosis. 


\section{INTRODUCCIÓN}

La cirrosis es el resultado final de enfermedades hepáticas crónicamente progresivas de diferentes etiologías (1). Fisiopatológicamente, la cirrosis es el resultado de un proceso de necrosis continua de los hepatocitos con pérdida del parénquima hepático, inflamación, fibrogénesis, cambios de regeneración celular y alteraciones de la macro y microcirculación, que conllevan a distorsión de la arquitectura hepática y formación de nódulos regenerativos (2-4). Por consiguiente, la cirrosis es entendida como un proceso dinámico, reversible en algún momento. En los últimos años se ha incentivado la investigación con el fin de adquirir nuevas herramientas e información que orienten el adecuado diagnóstico, manejo y enfoque de esta patología (5-7).

Clásicamente, el diagnóstico definitivo de cirrosis se establece con el explante del hígado cirrótico durante la cirugía de trasplante hepático; en una cirugía general, cuando se observa la superficie nodular del hígado; con la biopsia hepática, al identificar nódulos francos de regeneración separados por septos fibrosos; y recientemente con los métodos no invasivos basados en ecografía, dentro de ellos, la elastografía en tiempo real o Supersonic (Shear Wave Elastography, SWE) $(1,8,9)$. El Supersonic es una técnica de elastografía que tiene la capacidad de mapear y medir la rigidez hepática $(\mathrm{RH})$ al implementar un software en un sistema de ultrasonido, el Aixplorer ${ }^{\circledR}$, que proporciona una imagen bidimensional, cuantitativa y de color de la rigidez del tejido hepático en tiempo real (10). El Supersonic reemplaza en algunas ocasiones, la toma de la biopsia hepática y es útil para el seguimiento de la mayoría de pacientes con hepatopatías (11-14).

En Colombia, la prueba se ha introducido recientemente, y hasta el momento no se conocen estudios del comportamiento de esta en pacientes diagnosticados con cirrosis. Por tanto, el objetivo de este estudio fue evaluar el comportamiento de la elastografía en tiempo real en pacientes con cirrosis en un centro de atención de enfermedades hepáticas y digestivas en la ciudad de Bogotá.

\section{MATERIALES Y MÉTODOS}

Estudio piloto retrospectivo de una cohorte con pacientes cirróticos con diagnóstico comprobado por biopsia hepática, imágenes, clínica o una combinación de estas. Se realizó respectivamente la revisión de historias clínicas de los pacientes y la elastografía en tiempo real (Supersonic) entre 1 de junio y 31 de diciembre del 2016, en un centro de la ciudad de Bogotá. Se obtuvo consentimiento verbal de los sujetos incluidos. Los criterios de inclusión fueron:

- Examen físico compatible con cirrosis: estigmas de hepatopatía crónica, nevus arácneos o hígado duro a la palpación.
- Imágenes compatibles con cirrosis.

- Biopsia hepática compatible con cirrosis o informe de estadio 4, según escala METAVIR.

A todos los participantes incluidos en el estudio se les practicó la prueba de elastografía siguiendo el protocolo recomendado por el fabricante y utilizado en los estudios pivótales publicados de la siguiente manera:

- Los participantes asistieron en ayunas y las mediciones se realizaron en el lóbulo derecho del hígado, a través de los espacios intercostales con el sujeto acostado en posición supina y con el brazo derecho en la abducción máxima.

- La técnica se realizó con una sonda convexa de banda ancha (SC6-1). En el modo SWE se crean ondas de cizalladura o corte en el tejido a partir de la fuerza de radiación acústica generada por impulsos dados por el ultrasonido. La velocidad de la onda de corte se estimó por medio de una adquisición tipo Doppler (10). La velocidad de onda de corte se usó para calcular la rigidez de los tejidos mediante la fórmula $\mathrm{E}=\mathrm{pc} 2$, donde $\mathrm{E}$ es la elasticidad del tejido en kilopascal (kPs), p es la densidad del tejido (kg/ $\mathrm{m}^{3}$ ) y c es la velocidad de la onda de corte $(\mathrm{ms})$.

- Los datos de elasticidad se codificaron por colores mediante el software, creando un mapa bidimensional de rigidez tisular que se muestra en un recuadro o caja sobre la imagen en modo B convencional. Se estableció una región de interés (ROI) en el recuadro donde se realizó la medida de la rigidez hepática. Utilizamos un ROI promedio de $2 \mathrm{~cm}$ y un recuadro de $3,5 \times 2,5 \mathrm{~cm}$.

- Las mediciones se clasificaron como fallidas cuando no se obtuvo señal en el recuadro, o esta era mínima en todas las adquisiciones.

\section{ANÁLISIS ESTADÍSTICO}

Los análisis estadísticos se basaron en los datos obtenidos de mínimo 5 mediciones consecutivas equivalentes a 15 mediciones del Supersonic (debido a que la imagen en el recuadro es el resultado de aproximadamente tres imágenes promediadas en el tiempo).

La información se analizó de manera descriptiva mediante el cálculo de medidas de tendencia central y de dispersión para las variables cuantitativas y categóricas, en frecuencias absolutas y relativas para el total de muestra y por grupo según toma de biopsia. Se validó el supuesto de normalidad de los datos continuos por medio de la prueba de ShapiroWilks. Debido a que los datos no presentaron una distribución normal, la diferencia entre el grupo de biopsia se realizó mediante la prueba de comparación de medianas (prueba de Wilcoxon), y para la comparación entre grupos etarios con la prueba de Kruskal-Wallis. Todos los análisis se realizaron en el paquete estadístico STATA versión 13. 
En este estudio no se realizó ninguna intervención o modificación intencionada de las variables biológicas, fisiológicas, psicológicas o sociales de los individuos. Se condujo de acuerdo con los principios declarados en la XVIII Asamblea Médica Mundial (Helsinki, 1964).

\section{RESULTADOS}

Se incluyeron 65 pacientes diagnosticados con cirrosis, con una mediana de edad de 58 años (48-67), 40 mujeres $(61,5 \%)$ y 25 hombres $(38,5 \%)$. Todos los pacientes tenían diagnóstico clínico de cirrosis con base en el examen físico, laboratorios, imágenes y endoscopia. Adicionalmente, 32 pacientes tenían una biopsia hepática previa compatible con un estadio F4 de la escala METAVIR. Los datos generales de los pacientes y de laboratorio se muestran en la Tabla $\mathbf{1}$.

Al practicar la prueba a los 65 pacientes se encontró una mediana de 16,6 kilopascal (kPs), con un rango intercuartílico (RIQ) entre 13,9 a 19,5, y con un valor mínimo de 12 y un máximo de 30,5; sin una diferencia significativamente estadística entre hombres y mujeres (Figura 1). Al realizar la comparación de los valores de kPs entre las mujeres con y sin biopsia no se observan diferencias significativas estadísticas $(p=0,64)$. Este mismo comportamiento se advirtió en el grupo de hombres $(p=0,26)$. La comparación entre hombres y mujeres por grupo de biopsia se detalla en la Figura 2.

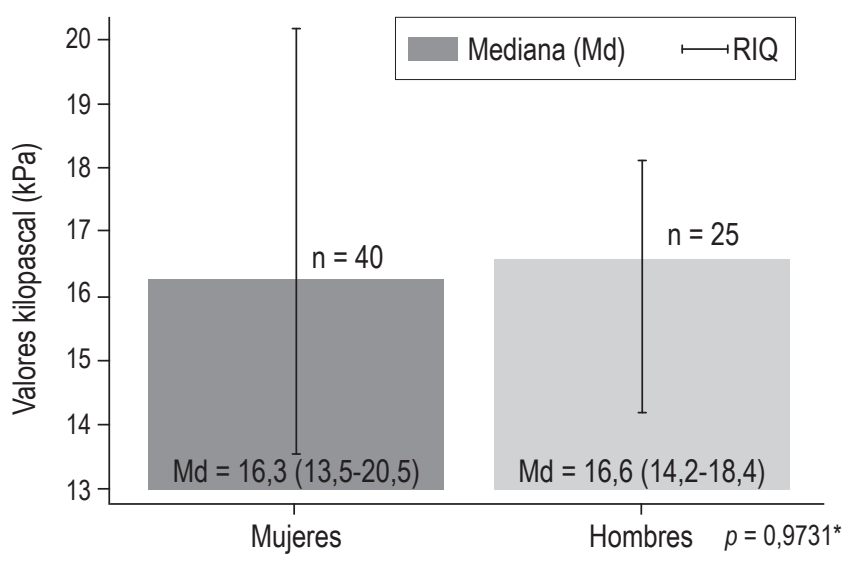

Figura 1. Valores de rigidez hepática según sexo. RIQ: rango intercuartílico. *Prueba Wilcoxon rank-sum.

En la Tabla 2 y Figura 3 se detallan los valores de rigidez por grupo etario con y sin biopsia. No se evidenciaron diferencias estadísticamente significativas.

Tabla 1. Pacientes diagnosticados con cirrosis por grupo con o sin biopsia

\begin{tabular}{|c|c|c|c|}
\hline & $\begin{array}{c}\text { Grupo con biopsia ( } n=32) \\
\text { Mediana (RIQ) }\end{array}$ & $\begin{array}{c}\text { Grupo sin biopsia }(n=33) \\
\text { Mediana (RIQ) }\end{array}$ & $\begin{array}{c}\text { Total }(n=65) \\
\text { Mediana (RIQ) }\end{array}$ \\
\hline Edad en años & $54,5(45,7-64)^{*}$ & $60(54-68)^{*}$ & $58(48-67)$ \\
\hline Índice de masa corporal (IMC) & $25(22-28,8)$ & $26(24-28)$ & $26(23-28)$ \\
\hline Leucocitos (cel/mL) & $5605(4440-6677)$ & $5430(4520-6625)$ & $5600(4460-6625)$ \\
\hline Hemoglobina (g/dL) & $14(13-15)$ & $15(13-16,5)$ & $14(13-16)$ \\
\hline Hematocrito \% & $43(39-46)$ & $44(40-49)$ & $44(40-47)$ \\
\hline Plaquetas (cel/mm³) & $161000(124750-226000)$ & $174000(125000-212500)$ & 168000 (125 500-219 000) \\
\hline Glucemia mg/dL & $95(86-101,7)^{*}$ & $105(91-116)^{*}$ & $97(89-112)$ \\
\hline AST UI/dL & $98,5(53,5-141,5)^{*}$ & $51(29-92)^{*}$ & $63(38-120)$ \\
\hline ALT UI/dL & $109,5(56,7-174,2)^{*}$ & $61(32-74,5)^{*}$ & $68(43-137)$ \\
\hline GGT UI/dL & $170,5(76,7-412,7)$ & $106(50,5-214,5)$ & $140(58,5-277)$ \\
\hline F alcalina UI/dL & $140(106,7-446,2)$ & $114(86,5-177)$ & $130(90,5-229,5)$ \\
\hline Bilirrubina total (mg/dL) & $1,04(0,7-1,6)$ & $0,8(0,45-1,25)$ & $0,9(0,6-1,4)$ \\
\hline Bilirrubina directa (mg/dL) & $0,45(0,3-0,8)^{*}$ & $0,3(0,2-0,6)^{*}$ & $0,4(0,2-0,7)$ \\
\hline Bilirrubina indirecta (mg/dL) & $0,5(0,4-0,77)$ & $0,5(0,2-0,65)$ & $0,5(0,31-0,7)$ \\
\hline Proteínas totales (g/dL) & $7,5(7-7,9)$ & $7,6(7,15-7,95)$ & $7,9(7,05-7,9)$ \\
\hline Albúmina (g/dL) & $4(3,6-4,3)$ & $4,1(3,7-4,5)$ & $4(3,7-4,4)$ \\
\hline
\end{tabular}

ALT: alanino aminotransferasa; AST: aspartato aminotransferasa; Cel: células; FA: fosfatasa alcalina; GGT: gamma glutamil transferasa; RIQ: rango intercuartílico.

${ }^{*}$ Diferencias estadísticamente significativas entre los dos grupos, $p<0,05$ (prueba de Wilcoxon Rank-sum). 
Tabla 2. Valores de rigidez (kPs) hepática por grupo etario, con o sin biopsia

\begin{tabular}{|c|c|c|c|c|c|c|}
\hline & \multicolumn{2}{|c|}{ Grupo con biopsia } & \multicolumn{2}{|c|}{ Grupo sin biopsia } & \multicolumn{2}{|c|}{ Total } \\
\hline & $n^{0}$ & Mediana (IQR) & $n^{0}$ & Mediana (IQR) & $\mathrm{n}^{0}$ & Mediana (IQR) \\
\hline Grupo etario & \multicolumn{2}{|c|}{$p=0,95^{\pi}$} & \multicolumn{2}{|c|}{$p=0,068 \pi$} & & \\
\hline $18-25$ & 3 & $16,5(12,8-24,7)$ & 1 & $14(14-14)$ & 4 & $15,3(13,1-22,6)$ \\
\hline $26-39$ & 4 & $14,9(12,5-18,9)$ & 1 & $13,3(13,3-13,3)$ & 5 & $13,3(12,6-18,3)$ \\
\hline $40-49$ & 6 & $16,6(13,6-20,0)$ & 2 & $12,5(12,1-13)$ & 8 & $15,2(13-18,1)$ \\
\hline $50-59$ & 8 & $17,6(13,8-21,1)$ & 12 & $16,9(14,6-23,2)$ & 20 & $16,9(14,6-22,7)$ \\
\hline $60-69$ & 7 & $15,6(13,5-18,7)$ & 10 & $16,4(14,8-21,2)$ & 17 & $16(14,1-19,1)$ \\
\hline$>70$ & 4 & $19(12,1-27,4)$ & 7 & $17,8(16,6-18,8)$ & 11 & $17,8(16,5-25,3)$ \\
\hline Total & 32 & $16,5(13,0-19,5)^{*}$ & 33 & $16,6(14,2-19,1)^{*}$ & 65 & $16,6(13,9-19,5)$ \\
\hline
\end{tabular}

${ }^{9}$ No hay diferencias significativas entre los grupos etarios con o sin biopsia (prueba Kruskal-Wallis).

${ }^{*} p=0,45$ no diferencias significativas entre los grupos (prueba Wilcoxon Rank-sum).
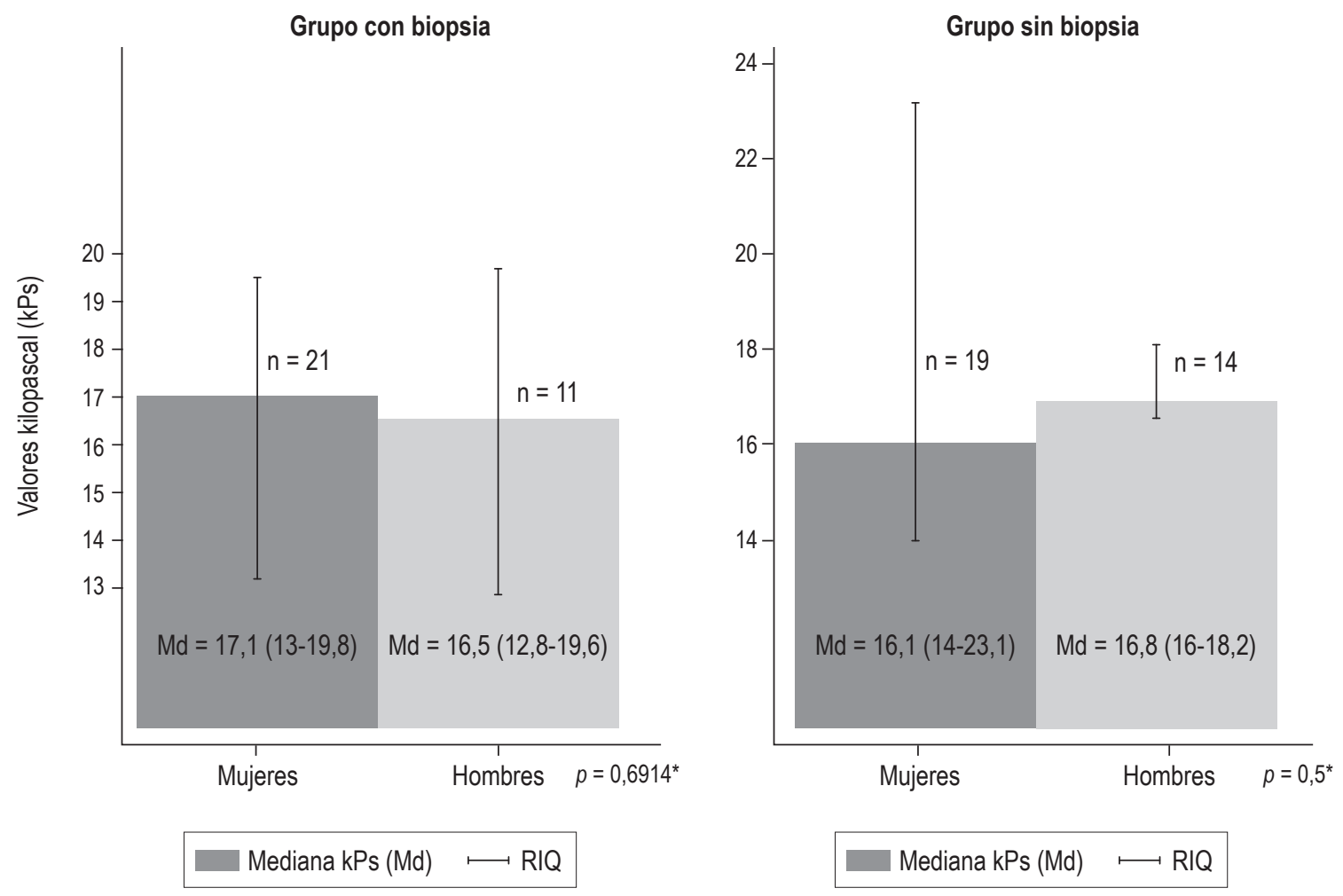

Figura 2. Valores de rigidez hepática según sexo. Grupos con y sin biopsia. RIQ: rango intercuartílico. *Prueba Wilcoxon rank-sum.

\section{DISCUSIÓN}

Conocer el grado de fibrosis hepática en un paciente es fundamental para la toma de decisiones con respecto a tratamientos y pronóstico de la enfermedad. En la hepatitis C crónica, la detección de fibrosis significativa es una indicación para el tratamiento antiviral inmediato y la detección de la cirrosis es una indicación para una monitorización más estricta del paciente en busca de las complicaciones relacionadas con la hipertensión portal, entre ellas hepatocarcinoma (HCC). Igualmente, en la hepatitis B, la fibrosis avanzada o la cirrosis son indicación de tratamiento de la enfermedad (15-17). En el hígado graso acontece lo mismo, aunque hay menos estudios $(18,19)$. 

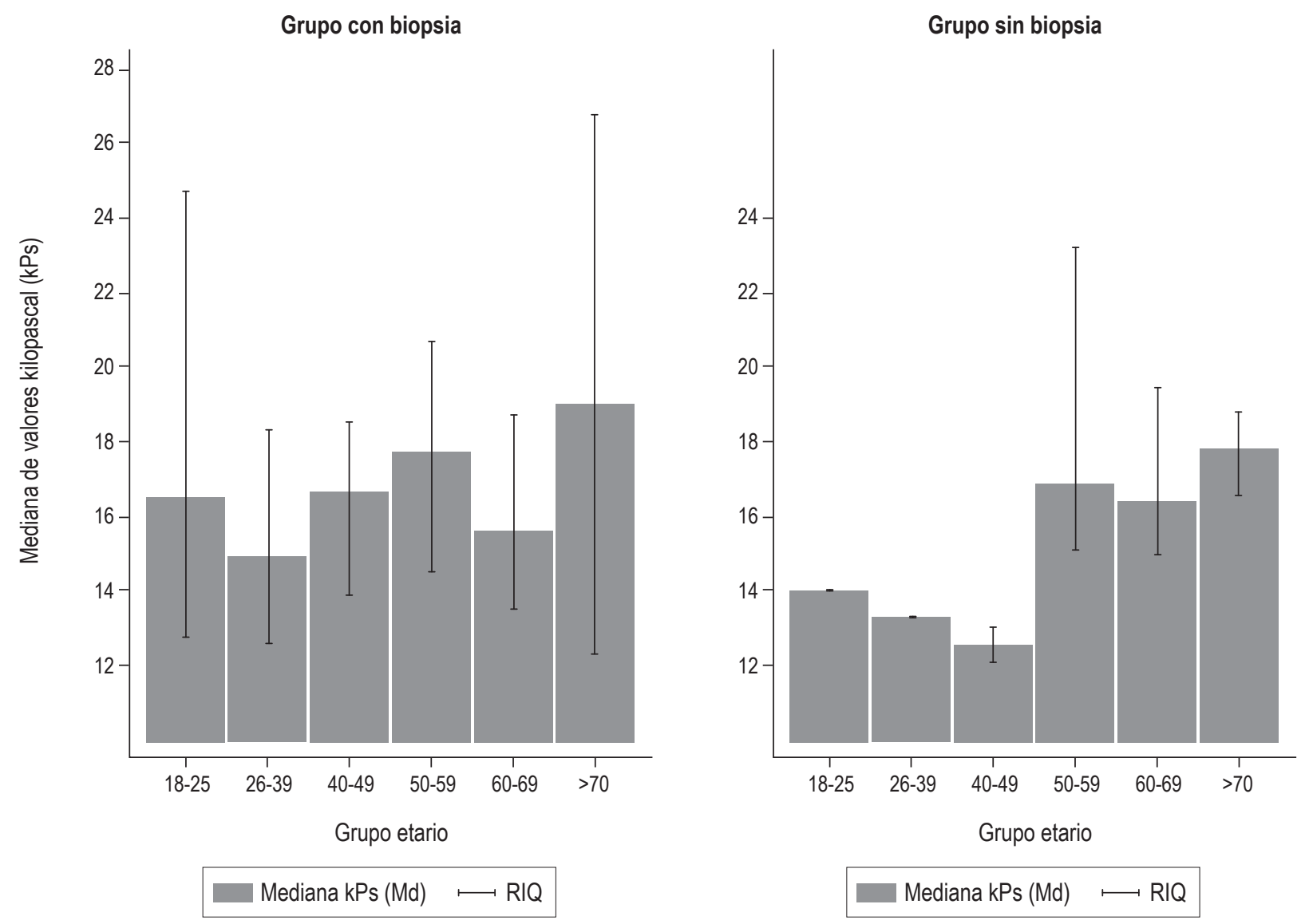

Figura 3. Valores de rigidez hepática según grupo etario por conjunto de biopsia. RIQ: rango intercuartílico.

Clásicamente, las pruebas no invasivas basadas en ecografía son excelentes en los extremos de la fibrosis, debido a la validez diagnóstica de la técnica con respecto al método de referencia (biopsia hepática) (9). En relación con la técnica del Supersonic, el estudio de Leung y colaboradores evaluó a 226 portadores de hepatitis B crónica, y demostró una mejor correlación con las puntuaciones de METAVIR de fibrosis para las mediciones de rigidez realizadas con SWE $(r=0,81)$ en comparación con TE $(r=0,58)(20)$.

Los puntos de corte calculados en el anterior estudio fueron consistentes con los encontrados en el estudio de Ziol y colaboradores en pacientes con hepatitis $C$ crónica: 6,5 kPs (METAVIR F $\geq 1$ ); 7,1 kPs (METAVIR F $\geq 2$ ); 7,9 kPs (METAVIR F $\geq 3$ ); y 10,1 kPs (METAVIR F4) (21). Un estudio realizado en el 2012 encontró un rendimiento en la identificación de fibrosis severa (mayor o igual de F3) y cirrosis (F4) muy alto. No se observaron diferencias significativas entre AUROC de TE y SWE en tiempo real para fibrosis severa $(0,96$ y 0,98 ; respectivamente $)$ y cirrosis $(0,96$ y 0,98 ; respectivamente), con puntos de corte para SWE de 8,7 y 10,4; respectivamente. La mediana para cirrosis fue de
15,6 con RIQ de 12,8 a 18,8; muy similar a la encontrada en nuestro estudio (16,6 kPs) (22). En el estudio anteriormente mencionado, los datos para cirrosis se basaron en 24 pacientes con F4 por biopsia, mediante la realización de la biopsia y pruebas no invasivas el mismo día. Este hecho representa una de las principales limitaciones en nuestro estudio, debido a que el tiempo entre la toma de biopsias y el Supersonic varía entre 1 semana y varios años.

En el estudio de Herman y colaboradores, las etiologías de la enfermedad hepática se categorizaron en infección crónica por hepatitis C $(\mathrm{VHC}, \mathrm{n}=379)$ o hepatitis $\mathrm{B}$ (VHB, $n=400)$, enfermedad de hígado graso no alcohólico (NAFLD, $\mathrm{n}=156)$ y un grupo de otras enfermedades hepáticas $(\mathrm{n}=199)$. Para el diagnóstico de fibrosis severa y cirrosis se encontró un punto de corte ligeramente inferior para los pacientes infectados con VHB ( $8,1 \mathrm{kPs}$ y $11,5 \mathrm{kPs}$, respectivamente) en comparación con todos los demás pacientes (9,2 kPs y $13,4 \mathrm{kPs}$, respectivamente). En general, el rendimiento diagnóstico fue mejor en pacientes con VHB y VHC que en pacientes sin hepatitis viral (23). En nuestro estudio, aunque conocemos la etiología, no la men- 
cionamos por el bajo número de pacientes. Son necesarios futuros estudios con un tamaño de muestra adecuado para realizar estos análisis por tipo de etiología.

En resumen, en estos 3 estudios previos vemos puntos de corte para cirrosis entre 10,1 y 13,4 kPs. En nuestro estudio, el punto de corte fue de 12 , y se presentaron 2 aspectos importantes, el primero es que no existió diferencia significativa entre pacientes con biopsia o sin ella, y el segundo es que los valores de medianas y RIQ son muy similares al estudio de Ferraioli y colaboradores (22).

En el medio, nuestro grupo publicó previamente un trabajo de elastografía en tiempo real en pacientes sanos, donde encontramos una media de 4,28 kPs; con una desviación estándar de 0,61. Este segundo trabajo junto con el anterior empiezan a dar elementos más objetivos de los valores normales y anormales en nuestros pacientes (24).

\section{CONCLUSIÓN}

Los valores de la rigidez hepática de los pacientes cirróticos analizados en este estudio piloto se encontraron dentro de los reportados en los estudios iniciales de las pruebas a nivel internacional, por lo que se podría sugerir la realización de esta prueba no invasiva en pacientes con sospecha de cirrosis, evitando así las complicaciones inherentes a la biopsia hepática, y convirtiéndose en una prueba muy útil en la práctica clínica.

\section{Agradecimientos}

Agradecemos a nuestros pacientes por habernos permitido ser nuestro objeto de estudio.

\section{Conflicto de intereses}

Los autores declaran no tener ningún conflicto de intereses.

\section{Fuentes de financiación}

Los autores declaran no tener ninguna fuente de financiación para la realización del estudio.

\section{REFERENCIAS}

1. Tsochatzis EA, Bosch J, Burroughs AK. Liver cirrhosis. Lancet. 2014;383(9930):1749-61. doi: 10.1016/S01406736(14)60121-5.

2. Garcia-Tsao G, Friedman S, Iredale J, Pinzani M. Now there are many (stages) where before there was one: in search of a pathophysiological classification of cirrhosis. Hepatology. 2010;51(4):1445-9. doi: http://doi.wiley.com/10.1002/ hep. 23478.
3. Lee UE, Friedman SL. Mechanisms of hepatic fibrogenesis. Best Pract Res Clin Gastroenterol. 2011;25(2):195-206. doi: https://doi.org/10.1016/j.bpg.2011.02.005.

4. Friedman SL. Mechanisms of hepatic fibrogenesis. Gastroenterology. 2008;134(6):1655-69. doi: https://doi. org/10.1053/j.gastro.2008.03.003.

5. Benvegnù L, Gios M, Boccato S, Alberti A. Natural history of compensated viral cirrhosis: a prospective study on the incidence and hierarchy of major complications. Gut. 2004;53(5):744-9. doi: https://doi.org/10.1136/ gut.2003.020263.

6. Friedman SL, Bansal MB. Reversal of hepatic fibrosis - Fact or fantasy? Hepatology. 2006;43(S1):S82-8. doi: https:// doi.org/10.1002/hep.20974.

7. Desmet VJ, Roskams T. Cirrhosis reversal: a duel between dogma and myth. J Hepatol. 2004;40(5):860-7. doi: https://doi.org/10.1016/j.jhep.2004.03.007.

8. Cassinotto C, Lapuyade B, Mouries A, Hiriart J-B, Vergniol $\mathrm{J}$, Gaye D, et al. Non-invasive assessment of liver fibrosis with impulse elastography: comparison of Supersonic Shear Imaging with ARFI and FibroScan ${ }^{\circledast}$ J Hepatol. 2014;61(3):550-7. doi: https://doi.org/10.1016/j. jhep.2014.04.044.

9. EASL-ALEH Clinical Practice Guidelines. Non-invasive tests for evaluation of liver disease severity and prognosis. J Hepatol. 2015;63(1):237-64. doi: https://doi. org/10.1016/j.jhep.2015.04.006.

10. Muller M, Gennisson JL, Deffieux T, Tanter M, Fink M. Quantitative viscoelasticity mapping of human liver using supersonic shear imaging: preliminary in vivo feasibility study. Ultrasound Med Biol. 2009;35(2):219-29. doi: https://doi.org/10.1016/j.ultrasmedbio.2008.08.018.

11. Samir AE, Dhyani M, Vij A, Bhan AK, Halpern EF, Méndez-Navarro J, et al. Shear-wave elastography for the estimation of liver fibrosis in chronic liver disease: determining accuracy and ideal site for measurement. Radiology. 2015;274(3):888-96. doi: https://doi.org/10.1148/ radiol.14140839.

12. Tada T, Kumada T, Toyoda H, Ito T, Sone $Y$, Okuda S, et al. Utility of real-time shear wave elastography for assessing liver fibrosis in patients with chronic hepatitis $\mathrm{C}$ infection without cirrhosis: comparison of liver fibrosis indices. Hepatol Res. 2015;45(10):E122-9. doi: https://doi. org/10.1111/hepr.12476.

13. Yoneda M, Thomas E, Sclair SN, Grant TT, Schiff ER. Supersonic shear imaging and transient elastography with the XL probe accurately detect fibrosis in overweight or obese patients with chronic liver disease. Clin Gastroenterol Hepatol. 2015;13(8):1502-9. doi: https:// doi.org/10.1016/j.cgh.2015.03.014.

14. Procopet B, Berzigotti A, Abraldes JG, Turon F, HernandezGea V, García-Pagán JC, et al. Real-time shear-wave elastography: applicability, reliability and accuracy for clinically significant portal hypertension. J Hepatol. 2015;62(5):1068-75. doi: https://doi.org/10.1016/j.jhep.2014.12.007. 
15. Ghany MG, Strader DB, Thomas DL, Seeff LB. Diagnosis, management, and treatment of hepatitis $C$ : an update. Hepatology. 2009;49(4):1335-74. doi: https://doi. org/10.1002/hep.22759.

16. European Association for the Study of the Liver. Recommendations on treatment of hepatitis C 2016. J Hepatol. 2018;66(1):153-94. doi: https://doi. org/10.1016/j.jhep.2016.09.001.

17. Sundaram V, Kowdley K. Management of chronic hepatitis B infection. BMJ. 2015;351(3):h4263. doi: https://doi. org/10.1136/bmj.h4263.

18. Haga Y, Kanda T, Sasaki R, Nakamura M, Nakamoto S, Yokosuka O. Nonalcoholic fatty liver disease and hepatic cirrhosis: comparison with viral hepatitis-associated steatosis. World J Gastroenterol. 2015;21(46):12989. doi: https://doi.org/10.3748/wjg.v21.i46.12989.

19. Farrell GC, Larter CZ. Nonalcoholic fatty liver disease: from steatosis to cirrhosis. Hepatology. 2006;43(S1):S99-112. doi: https://doi.org/10.1002/hep.20973.

20. Leung VY, Shen J, Wong VW, Abrigo J, Wong GL, Chim AM, et al. Quantitative elastography of liver fibrosis and spleen stiffness in chronic hepatitis b carriers: comparison of shear-wave elastography and transient elastography with liver biopsy correlation. Radiology. 2013;269(3):910-8. doi: https://doi.org/10.1148/radiol.13130128.

21. Ziol M, Handra-Luca A, Kettaneh A, Christidis C, Mal F, Kazemi F, et al. Noninvasive assessment of liver fibrosis by measurement of stiffness in patients with chronic hepatitis C. Hepatology. 2005;41(1):48-54. doi: https://doi. org/10.1002/hep.20506.

22. Ferraioli G, Tinelli C, Dal Bello B, Zicchetti M, Filice G, Filice C. Accuracy of real-time shear wave elastography for assessing liver fibrosis in chronic hepatitis C: a pilot study. Hepatology. 2012;56(6):2125-33. doi: https://doi. org/10.1002/hep. 25936 .

23. Herrmann E, de Lédinghen V, Cassinotto C, Chu WC, Leung VY, Ferraioli G, et al. Assessment of biopsy-proven liver fibrosis by two-dimensional shear wave elastography: an individual patient data-based meta-analysis. Hepatology. 2018;67(1):260-72. doi: https://doi.org/10.1002/ hep.29179.

24. Prieto Ortíz JE, Sánchez Pardo S, Prieto Ortíz RG, GarzónOrjuela N, Eslava-Schmalbach J. Elastografía en tiempo real (supersonic): comportamiento de la prueba en sujetos sanos de Bogotá. Rev Colomb Gastroenterol. 2017;32(4):332. 